\title{
Pflanzliche Extrakte gegen virale Infektionen des oberen Rachenraumes
}

\author{
Gibt es rationalisierbare protektive Möglichkeiten?
}

\author{
Andreas Hensel', Verena Spiegler', Karin Kraft ${ }^{2}$ \\ 1 Institut für Pharmazeutische Biologie und Phytochemie, Universität Münster \\ 2 Lehrstuhl für Naturheilkunde, Universitätsmedizin Rostock
}

Coronaviren sind seit wenigen Wochen in aller Munde, Influenzaviren seit vielen Jahrzehnten eine Plage, Rhinoviren und der jährliche Schnupfen, Adenoviren, RSVViren und Bronchialerkrankungen - Viruspartikel machen uns in der Tat das Leben schwer. Antivirale Medikationen sind in einigen Fällen durchaus möglich und auch als einschlägige Therapien etabliert (z.B. gegen Influenza), allerdings - und das ist die schlechte Nachricht - gegen die meisten viralen Pathogene im Bereich des Rachens und der oberen Luftwege verfügen wir nicht über spezifisch wirksame Substanzen. Das bedeutet jedoch nicht unbedingt, dass es keine Möglichkeiten gäbe, sich zu schützen. So verfügen die behüllten Viren (z. B. Corona-, RSV- oder Influenzaviren) über eine Hülle aus einer Lipiddoppelschicht, die sich aus zellulären Membransystemen der Wirtszelle ableitet. Diese Virushüllen sind durch viele exogene Stoffe (z. B. Lösungsmittel, Detergentien, lipophile Stoffe wie z. B. ätherische Öle) relativ leicht angreifbar. In diese Lipidschicht sind häufig auch (Glyco-) Proteine eingelagert, die exponiert herausragen können. Diese Proteine sind in vielen Fällen für die hochspezifische Erkennung der jeweiligen Wirtszellen, den damit verbundenen physikalischen Interaktionen, der nachfolgenden Adhäsion und dadurch letztlich auch für die Invasion in die Wirtszelle verantwortlich. Bekannte Beispiele hierfür sind das Haemagglutinin der Influenzaviren oder die S-Proteine der Coronaviren. Die typische Eintrittspforte solcher Erreger über eine Tröpfcheninfektion ist der Nasen-Rachen-Raum. Die nachfolgende Besiedlung der Epithelien erfolgt bei Influenzaviren eher im Bronchialbereich, während z.B. Covid-19-Infektionen durch Interaktion der viralen Hüllproteine mit komplementären Bindungsstrukturen im Rachenbereich manifest werden.

\section{Gerbstoffe}

Was spricht eigentlich dagegen, den Mund-RachenRaum gegen das Eindringen solcher Viruspartikel zu schützen? Behüllte Viruspartikel sind durch exogene Noxen angreifbar - wäre es denkbar, eine gewisse
Protektion durch Lokalbehandlung der Mundschleimhaut zu treffen? Im Bereich der Naturstoffe kennen wir einige Drogen, die große Mengen an Gerbstoffen (syn. Tannine) enthalten. Diese sehr heterogene Naturstoffgruppe beinhaltet die sog. Proanthocyanidine (syn. kondensierte Gerbstoffe), hydrolysierbare Gerbstoffe (syn. Gallotannine) und die sog. Lamiaceengerbstoffe (Depside). Allen ist gemeinsam, dass sie stark mit Proteinen interagieren und dadurch Proteinstruktur und -funktionalität teilweise erheblich verändern resp. inhibieren können. Tannine haben deshalb häufig unspezifische antimikrobielle Wirkungen, können aber auch durchaus die Funktionalität viraler Hüllproteine unterbinden. Aus etlichen Publikationen sind z. B. inhibierende Wirkungen von kondensierten Gerbstoffen resp. von gerbstoffhaltigen Extrakten bei Influenzaviren bekannt. Mechanistische Untersuchungen unter Verwendung eines gereinigten Proanthocyanidins (Procyanidin B2-3-O-digallat) zeigten, dass dieses Polyphenol relativ spezifisch die Bindungsdomäne von Haemagglutinin des Influenza-A-Virus hemmt, wodurch eine Adhäsion des Viruspartikels an die Wirtszelle nicht mehr möglich ist. Ähnliche Effekte wurden auch gegen Herpes-simplex-Viren (HSV-1) berichtet, wobei auch hier die entsprechenden Adhäsionsproteine in der Virushülle als Target benannt werden konnten. Derartige Proanthocyanidine sind im Arzneipflanzenbereich weit verbreitet (z. B. Grüntee, Sauerampferkraut, Cistrosenkraut etc.). Natürlich können diese Pflanzen nun nicht als Therapeutikum bei manifesten Virusinfektionen verwendet werden. Aber es wäre aus Sicht der Autoren durchaus denkbar, Darreichungsformen zur Anwendung zu bringen, die hochkonzentrierte Gerbstoffextrakte zur lokalen Anwendung in der Mundhöhle als Prophylaxe einsetzen. Was spricht z. B. gegen Sauerampferbonbons oder Kaugummis, die Proanthocyanidine in möglichst hoher Konzentration enthalten? Warum nicht auch Gurgel- und Mundspüllösungen anbieten, die solche Extrakte enthalten? Für regelmäßiges Gurgeln mit Grüntee bzw. Grüntee-Extrakt über mehrere Monate ergaben 5 kontrollierte (Wasser oder keine Intervention) 
Studien Hinweise auf eine präventive Wirkung gegenüber Influenzaviren.

Man könnte nun dagegen argumentieren, dass solche Prophylaxemethoden nur kurzzeitig Schutz bieten, solange das Bonbon gelutscht wird oder die Gurgellösung im Mund geschwenkt wird. Immerhin könnten diese Maßnahmen aber während einer möglichen Exposition oder unmittelbar danach eingesetzt werden.

Es gibt aber noch weitere mögliche Angriffspunkte, dies müsste allerdings durch gezielte Untersuchungen vor allem in vivo weiter belegt werden: Gerbstoffe interagieren auch mit Proteinbestandteilen in der Mundhöhle, z. B. Speichelproteinen und mit Oberflächenproteinen der Schleimhautepithelien. Jedem ist sicherlich das veränderte Mundgefühl nach Einnahme von gerbstoffhaltigen Zubereitungen (z. B. lang gezogener Schwarztee) bekannt. Im Prinzip ist dies auf Gerbstoffinteraktionen auf und mit den Epithelzellen zurückzuführen, wir „gerben“ also leicht die Buccalund Zungenoberfläche. Hierbei werden die Tannine natürlich für eine gewisse Zeit auf den Epithelien immobilisiert, sie sollten also länger und vielleicht auch retardiert vorliegen. Vielleicht könnte auch dies einen ankommenden Virus an der Adhäsion hemmen? Dies bleibt zu untersuchen, aber es bestehen gute Chancen, dass diese Hypothese stimmen könnte.

\section{Ätherische Öle}

Neben den Gerbstoffen scheinen auch bestimmte ätherische Öle antivirales Potenzial gegen behüllte Viren zu besitzen. Auch hier steht vor allem die Interaktion mit viralen Hüllproteinen im Vordergrund, die das Andocken, bzw. die Aufnahme der Viren in die Zelle verringert. Die meisten Untersuchungen wurden an Herpes-simplexViren (HSV-1 / HSV-2) durchgeführt, für 1,8-Cineol, dem Hauptbestandteil des Eukalyptusöls, wurden jedoch auch protektive Effekte in Mäusen beobachtet, die mit Influenzavirus C (IFV-C) infiziert waren. Dabei scheint es sich weniger um einen direkten antiviralen Effekt, sondern vielmehr um eine Verringerung der Entzündungsreaktionen zu handeln, die während der Pneumonie die Lunge betreffen. Ätherisches Öl ist jedoch nicht gleich ätherisches Öl. Aus aktuellem Anlass interessant ist die In-vitro-Aktivität von Lorbeeröl gegen Coronavirus SARSCoV: Das ätherische Öl aus den oberirdischen Teilen des Lorbeerbaumes (Laurus nobilis) inhibierte das Wachstum der Viren stärker als andere untersuchte ätherische Öle aus anderen Arten. Lorbeeröl enthält größere Anteile der Monoterpene 1,8-Cineol und $\beta$-Ocimen sowie des 
Sesquiterpens Dehydrocostuslacton. Inhalative Anwendungen von Lorbeeröl wären hier denkbar, das ggf. allergisierende Potenzial sollte aber bedacht werden. Einen schwächeren Effekt zeigte Salbeiöl, das ebenfalls reich an 1,8-Cineol ist. Neben ätherischem Öl enthalten Salbeiblätter (Salvia officinalis) außerdem kondensierte Gerbstoffe, sodass hier auch andere Zubereitungen wie Gurgellösungen sinnvoll eingesetzt werden könnten.

Warum also in Zeiten einer kaum beherrschbaren VirusPandemie nicht auch einmal unkonventionell etwas versuchen und testen? Warum nicht einfach Gerbstoff- oder Ätherischöl-haltige Lutschzubereitungen entwickeln oder entsprechende Gurgel- oder Spüllösungen probieren? Nicht als Arzneimittel - dafür benötigen wir behördliche Zulassungen, aber einfach als Lebensmittel?

Ein Versuch wäre es wert! Die präklinischen Daten aus vielen In-vitro-Prüfungen geben durchaus eine rationale und wissenschaftliche Grundlage. Wir dürfen uns dadurch nicht in Sicherheit wiegen, aber probieren kann man sicher!

Die Autoren wünschen Ihnen allen viel Gesundheit!

Interessenkonflikt

Die Autoren erklären, dass kein Interessenkonflikte besteht.

Korrespondenzadresse

Prof. Dr. Andreas Hensel

Dr. Verena Spiegler

Universität Münster

Institut für Pharmazeutische Biologie und Phytochemie

Corrensstr. 48

48149 Münster

E-Mail: ahensel@uni-muenster.de

Prof Dr. med. Karin Kraft

Lehrstuhl für Naturheilkunde

Universitätsmedizin Rostock

Ernst-Heydemannstr. 6

18057 Rostock

E-Mail: karin.kraft@med.uni-rostock.de

\section{Literatur}

[1] Brandão E, Silva MS, García-Estévez I et al. Inhibition mechanisms of wine polysaccharides on salivary protein precipitation. J Agric Food Chem 2020; 11: 2955-2963. doi: 10.1021/ acs.jafc.9b06184

[2] Derksen A, Hensel A, Hafezi W et al. 3-O-galloylated procyanidins from Rumex acetosa $\mathrm{L}$. inhibit the attachment of influenza A virus. PLoS One 2014; 9(10):e110089. doi: 10.1371/ journal.pone.0110089

[3] Gescher K, Kühn J, Lorentzen E et al. Proanthocyanidin-enriched extract from Myrothamnus flabellifolia Welw. exerts antiviral activity against Herpes simplex virus type 1 by inhibition of viral adsorption and penetration. J Ethnopharmacol 2011; 134: 486-474. doi: 10.1016/j.jep.2010.12.038

[4] Gescher K, Hensel A, Hafezi W et al. Oligomeric proanthocyanidins from Rumex acetosa $\mathrm{L}$. inhibit the attachment of Herpes simplex virus type-1. Antiviral Res 2011; 89: 9-18. doi:10.1016/j.antiviral.2010.10.007

[5] Ide K, Yamada H, Kawasaki Y et al. Effect of gargling with tea and ingredients of tea on the prevention of influenza infection: a meta-analysis BMC Public Health 2016; 16: 396. doi:10.1186/s12889-016-3083-0

[6] Kim SJ, Lee JW, Eun YG et al. Pretreatment with a grape seed proanthocyanidin extract downregulates proinflammatory cytokine expression in airway epithelial cells infected with respiratory syncytial virus. Mol Med Report 2019; 19: $3330-3336$

[7] Li Y, Lai Y, Wang Y et al. 1, 8-Cineol protect against influenzavirus-induced pneumonia in mice. Inflammation 2016; 39 : 1582-1593

[8] Loizzo MR, Saab AM, Tundis R et al. Phytochemical analysis and in vitro antiviral activities of the essential oils of seven Lebanon species. Chem Biodivers 2008; 5: 461-470

[9] Reichling J, Schnitzler P, Suschke U, Saller R. Essential oils of aromatic plants with antibacterial, antifungal, antiviral, and cytotoxic properties - an overview. Forsch Komplementmed 2009; 16: 79 - 90. doi: 10.1159/000207196

[10] Soares S, Brandão E, Garcia-Estevez I et al. Interaction between ellagitannins and salivary proline-rich proteins. J Agric Food Chem 2019; 67: 9579-9590. doi: 10.1021/acs. jafc.9b02574

Bibliografie

DOI https://doi.org/10.1055/a-1102-1662

Zeitschrift für Phytotherapie 2020; 41: 52-54

(c) Georg Thieme Verlag KG Stuttgart · New York ISSN 0722-348X 